\title{
Pediatric radiologists and burnout: identifying stressors and moving forward
}

\author{
Michael J. Callahan ${ }^{1}$ \\ Received: 25 March 2019 / Accepted: 28 March 2019 /Published online: 8 May 2018 \\ (C) Springer-Verlag GmbH Germany, part of Springer Nature 2019
}

"Pediatric imaging is fun. It is exciting and stimulating, and I would not trade subspecialties with anyone" [1]. These are the inspiring words of the late Tom Slovis, MD. Dr. Slovis was a giant in the field of pediatric radiology and a mentor to a generation of pediatric radiologists [2]. Nearly 20 years ago, he described an ongoing set of changes in the practice of pediatric radiology [1], and related many of these changes to advances in technology. Slovis astutely referred to a book by John Naisbitt et al. [3] titled High Tech High Touch: Technology and Our Search for Meaning, which submits that "Americans are intoxicated by technology ... at its best, technology supports and improves human life and ... at its worst, it alienates isolates, distorts, and destroys."

Advances in imaging technology and picture archiving and communication system (PACS) technology are essential for the effective practice of radiology in the 21 st century. Although such technological advances generally lead to increased efficiency, one potential consequence of digitization is increased isolation and depersonalization, which can serve as potential sources of stress, alienation and burnout, particularly for radiologists [4].

The topic of burnout and disengagement in radiologists has become a major focus of the American College of Radiology (ACR). At the 2018 Intersociety Summer Conference (ISC) in Stowe, VT, leaders from the ACR and societal leaders from numerous imaging subspecialties reviewed the "current state of stress and burnout in our workplaces, and identified approaches to foster engagement, wellness, and job satisfaction" [5]. The principal goal of the 2018 ISC was to identify strategies and themes to mitigate the frequency, manifestations and impact of stress and burnout [5].

Michael J. Callahan

michael.callahan@childrens.harvard.edu

1 Department of Radiology,

Boston Children's Hospital,

300 Longwood Ave.,

Boston, MA 02115, USA
The triple aim of health care, according to the Institute for Healthcare Improvement, includes improving the patient experience of care, improving the health of populations, and reducing the per capita cost of health care [6]. Kruskal et al. [5] contended that a fourth aim of health care "implores physicians to take care of themselves and their own wellness" and found that such discussions must be continued over an extended period until solutions are reached.

Dr. Ayyala and colleagues [7] took on the important task of identifying the prevalence of stress and burnout among a sample of Society for Pediatric Radiology (SPR) members by means of an anonymous survey. Ayyala et al. [7] found that the prevalence of emotional exhaustion and depersonalization was $66 \%$ and $61 \%$, respectively, and the prevalence of a perceived lack of personal accomplishment was present in $15 \%$ of responders. This relatively high incidence might be in part related to sample bias, but the findings are striking and concerning nonetheless. Key stressors that contribute to depersonalization and burnout in pediatric radiology include a work-life imbalance, significant call burden, lower rates of reimbursement, and fewer years of experience [7]. Perhaps an increase in work volumes, simultaneous coverage of multiple modalities and multiple imaging sites, and diminishing interactions with fellow pediatric radiologists, our clinical colleagues and our patients represent other potential sources of stress and burnout.

Clearly, physician stress and burnout is an extremely important matter of discussion for pediatric radiologists, and Ayyala et al. [7] should be commended for initiating the dialogue. Indeed, this topic was a major focus at the SPR members' forum at the 2018 Radiological Society of North America meeting, and these important issues were recently discussed at a 2019 SPR Board of Directors retreat in Denver, CO. Potential solutions discussed at these venues included, but were not limited to, education of hospital leaders on the importance of the topic of physician stress and burnout, fostering flexibility in physician scheduling, and leveraging the advantages of artificial intelligence to improve efficiency for diagnostic imaging 
interpretation. Although theoretical solutions exist for a multitude of problems, steps to moderate and manage the effects and consequences of burnout in pediatric radiologists will undoubtedly require in-depth discussions at future SPR meetings.

According to Kruskal et al. [5], potential strategies to mitigate the effects of stress and burnout for radiologists include minimizing the stigma of burnout, reducing radiologist isolation through building community and fostering connectivity, utilizing data and benchmarking to guide effectiveness of improvement efforts, resourcing and training "wellness" committees, acknowledging value contributions of team members, and improving efficiency in the workplace. Further work is necessary to identify and institute effective global and personal solutions for all radiologists.

Ayyala et al. [7] identified that stress and burnout are real problems for many members of the SPR, but this topic is certainly not specific to pediatric radiology. According to Kruskal et al. [5], a 2018 ACR Commission on Human Resources workforce survey demonstrated that $78 \%$ of radiologists, midlevel providers, and physicists reported burnout being a significant problem in their workplace. Only $19 \%$ reported mechanisms for assessing it, and only $21 \%$ said they had effective ways of addressing the problem [5]. Nevertheless, pediatric radiologists must work together in an effort to moderate these issues before they become more prevalent and potentially even more problematic.

What should be the next steps? Does this topic need to be formally addressed by the SPR leadership? Or should issues related to stress and burnout be addressed at an institutional level, or a departmental/divisional level? Ultimately, does the burden fall on the individual physician? Unfortunately, there are no simple solutions to this multifaceted topic, but initiating the discussion and acknowledging the importance of these issues provides an important starting point.

Fostering tangible solutions for mitigating stress and burnout will be necessary going forward, but it is also critically important for all pediatric radiologists to recall the immense benefits of practicing in our subspecialty - benefits that are not necessarily shared by other imaging subspecialties.

Dr. Gunderman [8] provided an eloquent summary of the unparalleled virtues of practicing pediatric radiology in a manuscript titled "The Lure of Pediatric Radiology." I would urge any pediatric radiologist who is suffering from the effects of stress and burnout to read this commentary, including these words: "The great pediatric radiologists know that caring for children is profoundly good for the soul. It reminds us that our lives have purposes that extend far beyond the prices of our possessions ... It brings out the best of the compassion, understanding, and courage we have in us."

Stress and burnout represent a growing problem for radiologists [5], including pediatric radiologists [7]. We must work together as a Society to provide support to those in need, promote and embrace effective solutions for this important problem, and all the while maintain the high level of care that we owe to our pediatric patients and their families.

\section{Compliance with ethical standards}

Conflicts of interest None

\section{References}

1. Slovis TL (2000) New horizons in pediatric radiology. Radiology 216:317-320

2. Joshi A, Strouse PJ (2018) Thomas L. Slovis. Pediatr Radiol 48:754-757

3. Naisbitt J, Naisbitt N, Phillips D (2001) High tech high touch: technology and our search for meaning. Nicholas Brealey Publishing, London

4. Salber J (2016) How technology squeezed the joy out of radiology. The Doctor Weighs In. https://thedoctorweighsin.com/howtechnology-squeezed-the-joy-out-of-radiology/. Accessed 25 March 2019

5. Kruskal JB, Shanafelt T, Eby P et al (2018) A road map to foster wellness and engagement in our workplace - a report of the 2018 summer intersociety meeting. J Am Coll Radiol. https://doi.org/10. 1016/j.jacr.2018.10.025

6. Lewis N (2014) A primer on defining the triple aim. Institute for Healthcare Improvement. http://www.ihi.org/communities/blogs/aprimer-on-defining-the-triple-aim. Accessed 25 March 2019

7. Ayyala RS, Ahmed FS, Ruzal-Shapiro C, Taylor GA (2019) Stressors contributing to burnout amongst pediatric radiologists: results from a survey of the Society for Pediatric Radiology. Pediatr Radiol. https://doi.org/10.1007/s00247-019-04370-z

8. Gunderman RB (2001) The lure of pediatric radiology. AJR Am J Roentgenol 176:1371-1373

Publisher's note Springer Nature remains neutral with regard to jurisdictional claims in published maps and institutional affiliations. 